\title{
APPROXIMATE SOURCE CONDITIONS IN TIKHONOV REGULARIZATION - NEW ANALYTICAL RESULTS AND SOME NUMERICAL STUDIES
}

\author{
B. HOFMANN ${ }^{1}$, D. DÜVELMEYER ${ }^{1}$ and K. KRUMBIEGEL ${ }^{2}$ \\ ${ }^{1}$ Chemnitz University of Technology, Department of Mathematics \\ Reichenhainer Str. 41, D-09107 Chemnitz, Germany \\ E-mail: hofmannb@mathematik.tu-chemnitz.de \\ E-mail: dana.duevelmeyer@mathematik.tu-chemnitz.de
}

${ }^{2}$ Johann Radon Institute for Computational and Applied Mathematics

Altenbergerstr. 69, A-4040 Linz, Austria

E-mail: klaus.krumbiegel@oeaw.ac.at

Received November 3, 2005; revised January 23, 2006

\begin{abstract}
We present some new ideas and results for finding convergence rates in Tikhonov regularization for ill-posed linear inverse problems with compact and non-compact forward operators based on the consideration of approximate source conditions and corresponding distance functions. The new results and studies complement and extend in numerous points the recent papers $[5,7,8,10]$ that also exploit the distance functions originally introduced in [2] which measure the violation of a moderate source condition that works as a benchmark. In this context, we distinguish as in [8] logarithmic, power and exponential decay rates for the distance functions and their consequences. Under specific range inclusions the decay rate of distance functions is verified explicitly, whereas in [10] this result is also used but formulated only in an implicit manner. Applications to non-compact multiplication operators are briefly reviewed from [8]. An important new result is that we can show for compact operators a one-to-one correspondence between the maximal power type decay rates for the distance functions and maximal exponents of Hölder rates in Tikhonov regularization linked by the specific singular value expansion of the solution element. Some numerical studies on simple integration illustrate the compact operator case and the specific situation of discretized problems. Finally, some ideas of generalization are mentioned concerning the fact that the benchmark of the distance function can be shifted.

Key words: linear ill-posed problems, Tikhonov regularization, approximate source conditions, distance functions, convergence rates, compact operator, singular value asymptotics, multiplication operators, range inclusions
\end{abstract}




\section{Introduction}

Let $X$ and $Y$ be infinite dimensional Hilbert spaces, where the symbol $\|\cdot\|$ denotes the generic norms in both spaces as well as associated operator norms. Moreover, $\langle\cdot, \cdot\rangle$ designates the inner product in Hilbert space. In this paper we are going to study ill-posed linear operator equations

$$
A x=y \quad(x \in X, y \in Y)
$$

with injective and bounded linear operators $A: X \rightarrow Y$ having a non-closed range $\mathcal{R}(A)$, for which the stable approximate solution requires regularization methods. In the sequel we focus on the Tikhonov method (see, e.g., [1, 2, 4], $[6,21])$ as a standard approach. Let $x_{0} \in X$ be the unique solution of equation (1.1) for an exact right-hand side $y=A x_{0} \in Y$. Instead of $y$ we assume to know the noisy data element $y^{\delta} \in Y$ with noise level $\delta>0$ and

$$
\left\|y^{\delta}-y\right\| \leq \delta .
$$

We distinguish regularized solutions

$$
x_{\alpha}=\left(A^{*} A+\alpha I\right)^{-1} A^{*} y
$$

with regularization parameter $\alpha>0$ in the case of noise-free data and

$$
x_{\alpha}^{\delta}=\left(A^{*} A+\alpha I\right)^{-1} A^{*} y^{\delta}
$$

in the case of noisy data.

Here we focus on the noise-free error function

$$
f(\alpha):=\left\|x_{\alpha}-x_{0}\right\|=\left\|\alpha\left(A^{*} A+\alpha I\right)^{-1} x_{0}\right\| \quad(\alpha>0)
$$

for fixed $A$ and $x_{0}$. Taking into account the noise level $\delta$ this function determines the total regularization error of the Tikhonov regularization

$$
e(\alpha, \delta):=\left\|x_{\alpha}^{\delta}-x_{0}\right\| \leq\left\|x_{\alpha}-x_{0}\right\|+\left\|x_{\alpha}^{\delta}-x_{\alpha}\right\|
$$

in case of noisy data with the well-known estimate

$$
e(\alpha, \delta) \leq f(\alpha)+\left\|\left(A^{*} A+\alpha I\right)^{-1} A^{*}\left(y^{\delta}-y\right)\right\| \leq f(\alpha)+\frac{\delta}{2 \sqrt{\alpha}} .
$$

\section{Convergence Rates for Tikhonov Regularization Based on Approximate Source Conditions}

To obtain convergence rates for the Tikhonov regularization and other linear regularization methods, in recent years general source conditions

$$
x_{0}=\varphi\left(A^{*} A\right) w \quad(w \in X)
$$


with index functions $\varphi(t)\left(0 \leq t \leq \bar{t}:=\|A\|^{2}\right)$ were used (see $[11,15,18]$ and [20]). In the sense of [15] we call $\varphi$ an index function if this function is continuous and strictly increasing with $\varphi(0)=0$. For the Tikhonov regularization method we search for estimates of the error function of form

$$
f(\alpha)=\left\|\alpha\left(A^{*} A+\alpha I\right)^{-1} \varphi\left(A^{*} A\right) w\right\| \leq K \varphi(\alpha)\|w\| \quad(0<\alpha \leq \bar{t})
$$

with some $K \geq 1$, which imply estimates of form

$$
e(\alpha, \delta) \leq K \varphi(\alpha)\|w\|+\frac{\delta}{2 \sqrt{\alpha}} \quad(0<\alpha \leq \bar{t})
$$

for the total regularization error. Then the following proposition can be obtained from the literature (see, e.g., $[3,15,16])$. In this context, we should note that a real function $f$ defined on $[0, \bar{t}]$ is said to be operator monotone if for two selfadjoint bounded linear operators $S_{1}$ and $S_{2}$ mapping in $X$ with spectrum in $[0, \bar{t}]$ the semi-ordering $S_{1} \leq S_{2}$, which is defined as $\left\langle S_{1} x, x\right\rangle \leq\left\langle S_{2} x, x\right\rangle$ for all $x \in X$, implies $f\left(S_{1}\right) \leq f\left(S_{2}\right)$. The use of operator monotone functions in connection with the analysis of ill-posed problems is due to [14].

Proposition 1. We assume that (2.1) holds and $\varphi(t)(0 \leq t \leq \bar{t})$ is an index function. If (a) $\varphi(t) / t$ is monotonically decreasing on $(0, \bar{t}]$, or (b) $\varphi(t)$ is concave on $[0, \bar{t}]$, then $(2.2)$ holds with $K=1$. If $(c) \varphi(t)$ is operator monotone on $[0, \bar{t}]$, then $(2.2)$ is valid with some $K \geq 1$. If there exists a $\hat{t} \in(0, \bar{t})$ such that $(d) \varphi(t) / t$ is monotonically decreasing on $(0, \hat{t}]$ or $(e) \varphi(t)$ is concave on $[0, \hat{t}]$, then $(2.2)$ is true with $K=\varphi(\bar{t}) / \varphi(\hat{t})$.

Avoiding the use of explicit general source conditions (2.1) this paper presents an alternative approach for finding estimates of the form (2.2) and hence (2.3) and consequently convergence rates for the Tikhonov regularization on the basis of the following lemma. We extend and complement in numerous points the corresponding results on this topic, which were published by the first author and coauthors in the recent papers [5, 7, 8] and [10].

Lemma 1. Based on the distance function

$$
d(R):=\inf \left\{\left\|x_{0}-A^{*} v\right\|: v \in Y,\|v\| \leq R\right\}
$$

that measures for $x_{0}$ the violation of the specific source condition

$$
x_{0}=A^{*} v_{0} \quad\left(v_{0} \in Y,\left\|v_{0}\right\| \leq R\right),
$$

we obtain

$$
f(\alpha)=\left\|x_{\alpha}-x_{0}\right\| \leq d(R)+\frac{\sqrt{\alpha}}{2} R
$$

for all $\alpha>0$ and $R \geq 0$ as an estimate for the error function of regularized solutions in Tikhonov regularization in the case of noise-free data.

Proof. Let $v \in Y$ with $\|v\| \leq R$. Then based on formula (1.2) we can estimate $f(\alpha)$ by the triangle inequality as follows: 


$$
\begin{aligned}
f(\alpha) & =\left\|\alpha\left(A^{*} A+\alpha I\right)^{-1} x_{0}-\alpha\left(A^{*} A+\alpha I\right)^{-1} A^{*} v+\alpha\left(A^{*} A+\alpha I\right)^{-1} A^{*} v\right\| \\
& \leq\left\|\alpha\left(A^{*} A+\alpha I\right)^{-1}\left(x_{0}-A^{*} v\right)\right\|+\left\|\alpha\left(A^{*} A+\alpha I\right)^{-1} A^{*} v\right\| \\
& \leq \alpha\left\|\left(A^{*} A+\alpha I\right)^{-1}\right\|\left\|x_{0}-A^{*} v\right\|+\alpha\left\|\left(A^{*} A+\alpha I\right)^{-1} A^{*}\right\|\|v\| \\
& \leq \alpha \frac{1}{\alpha}\left\|x_{0}-A^{*} v\right\|+\alpha \frac{1}{2 \sqrt{\alpha}}\|v\| \leq\left\|x_{0}-A^{*} v\right\|+\frac{1}{2} \sqrt{\alpha} R .
\end{aligned}
$$

Since the inequality

$$
f(\alpha) \leq\left\|x_{0}-A^{*} v\right\|+\frac{1}{2} \sqrt{\alpha} R
$$

also remains valid if we substitute $\left\|x_{0}-A^{*} v\right\|$ by

$$
\inf \left\{\left\|x_{0}-A^{*} v\right\|: v \in Y,\|v\| \leq R\right\},
$$

we immediately find the inequality (2.6). This proves the lemma.

Note that an estimate

$$
f(\alpha) \leq \sqrt{d^{2}(R)+\alpha R^{2}}
$$

implying the inequality

$$
f(\alpha) \leq d(R)+\sqrt{\alpha} R,
$$

which was already used in the papers $[5,8,10]$, had been originally shown in [2, Theorem 6.8, p.97-98]. Compared to (2.7) our formula (2.6) has a smaller constant. On the other hand, the proof of Lemma 1 is much easier than Baumeister's proof in [2]. Therefore, we have presented the proof in this section, although Lemma 1 is only a corollary of Lemma 2 from Section 6 .

Evidently, for every $x_{0} \in X$ the nonnegative distance function $d(R)$ from (2.4) depending on the radius $R \in[0, \infty)$ is well-defined and non-increasing with $\lim _{R \rightarrow \infty} d(R)=0$ as a consequence of the injectivity of $A$ and $\overline{\mathcal{R}\left(A^{*}\right)}=X$. The distance function $d(R)$ expresses the behaviour of $x_{0}$ with respect to the benchmark condition (2.5) which can be rewritten as $x_{0}=\left(A^{*} A\right)^{\frac{1}{2}} w(w \in X)$ and which is moderate within the family of source conditions (2.1) of powertype $\varphi(t)=t^{\eta}$ for exponents $0<\eta \leq 1$.

Example 1 [Logarithmic type decay]. If $d(R)$ decreases to zero very slowly as $R \rightarrow \infty$, the resulting rate for $f(\alpha) \rightarrow 0$ as $\alpha \rightarrow 0$ is also very slow. Here, we consider the family of distance functions

$$
d(R) \leq K(\ln R)^{-p} \quad(\underline{R} \leq R<\infty)
$$

for some constants $\underline{R}>0, K>0$ and for parameters $p>0$. By setting

$$
R=R(\alpha):=\alpha^{-\kappa} \quad\left(0<\kappa<\frac{1}{2}\right)
$$


and taking into account that $\alpha=o\left((\ln (1 / \alpha))^{-p}\right)$ as $\alpha \rightarrow 0$ we have from Lemma 1 and (2.8)

$$
f(\alpha) \leq \widetilde{K}(\ln (1 / \alpha))^{-p} \quad(0<\alpha \leq \bar{\alpha})
$$

for some $\bar{\alpha}>0$ and a constant $\widetilde{K}>0$. Then by using the a priori parameter choice $\alpha(\delta)=c_{0} \delta^{\chi}$ with some exponent $0<\chi<2$ we obtain the logarithmic convergence rate

$$
e(\alpha(\delta), \delta)=\mathcal{O}\left((\ln (1 / \delta))^{-p}\right) \quad \text { as } \quad \delta \rightarrow 0
$$

discussed, e.g., in [11] with respect to general source conditions (2.1) and corresponding logarithmic index functions $\varphi$.

Example 2 [Power type decay]. If $d(R)$ behaves as a power of $R$, i.e.,

$$
d(R) \leq K / R^{\frac{\gamma}{1-\gamma}} \quad(\underline{R} \leq R<\infty)
$$

with parameters $0<\gamma<1$ and constants $\underline{R}>0, K>0$, then by setting $R=R(\alpha):=\alpha^{\frac{\gamma-1}{2}}$ we derive from Lemma 1 an estimate

$$
f(\alpha) \leq \widetilde{K} \alpha^{\frac{\gamma}{2}} \quad(0<\alpha \leq \bar{\alpha})
$$

for some $\bar{\alpha}>0$ and a constant $\widetilde{K}>0$. Here, the exponent in (2.11) attains all positive real numbers when $\gamma$ covers the open interval $(0,1)$. If the a priori parameter choice $\alpha(\delta) \sim \delta^{\frac{2}{1+\gamma}}$ is used, we find from (1.4) and (2.12)

$$
e(\alpha(\delta), \delta)=\mathcal{O}\left(\delta^{\frac{\gamma}{1+\gamma}}\right) \quad \text { as } \quad \delta \rightarrow 0 .
$$

For $0<\gamma<1$ formula (2.13) includes all Hölder convergence rates that are slower than the rate $\mathcal{O}(\sqrt{\delta})$ which characterizes the source condition (2.5).

Example 3 [Exponential type decay]. Even if $d(R)$ falls exponentially, i.e.,

$$
d(R) \leq K \exp \left(-c R^{q}\right) \quad(\underline{R} \leq R<\infty)
$$

for parameters $q>0$ and constants $\underline{R}>0, K>0$ and $c \geq \frac{1}{2}$, the convergence rate $\mathcal{O}(\sqrt{\delta})$ cannot be obtained on the basis of Lemma 1. From (2.6) we have with $R=R(\alpha):=(\ln (1 / \alpha))^{1 / q}$ the estimate

$$
f(\alpha) \leq \widetilde{K}(\ln (1 / \alpha))^{1 / q} \sqrt{\alpha} \quad(0<\alpha \leq \bar{\alpha})
$$

for some $\bar{\alpha}>0$ and a constant $\widetilde{K}>0$. Hence with $\alpha(\delta) \sim \delta$ we derive a convergence rate

$$
e(\alpha(\delta), \delta)=\mathcal{O}\left((\ln (1 / \delta))^{1 / q} \sqrt{\delta}\right) \quad \text { as } \quad \delta \rightarrow 0,
$$

which is only a little slower than $\mathcal{O}(\sqrt{\delta})$.

At the end of this section we should note that a modified variant of distance function (2.4) with a graph norm $\|\cdot\|_{*}$ instead of the generic Hilbert space norm $\|\cdot\|$ was already exploited for an error analysis in [13]. 


\section{An Application Based on Range Inclusions}

In paper [10] the situations of Examples 1 and 2 in Section 2 apply to specific linear operators $A$ with non-closed range, which are forward operators in inverse PDE problems. The theoretic background to those applications is formulated by the following proposition based on range inclusions. The estimate (3.2) of this proposition helps to characterize the decay rate of $d(R) \rightarrow 0$ as $R \rightarrow \infty$ under the formulated assumptions.

Proposition 2. We assume that the solution smoothness of the operator equation (1.1) is characterized by a condition

$$
x_{0}=G w \quad(w \in X),
$$

where $G: X \rightarrow X$ is an injective, compact, positive self-adjoint, linear operator. Moreover, we assume that there exists an index function $\varrho(t)(0 \leq t \leq$ $\|G\|)$ such that

$$
\mathcal{R}(\varrho(G)) \subset \mathcal{R}\left(\left(A^{*} A\right)^{\frac{1}{2}}\right)=\mathcal{R}\left(A^{*}\right) .
$$

We set $\psi(t)=t / \varrho(t)(0<t \leq\|G\|)$ and suppose that $\psi$ is strictly decreasing on the interval $(0, \varepsilon]$ for some small positive value $\varepsilon$ and fulfills the limit condition $\lim _{t \rightarrow 0} \psi(t)=\infty$. Then we have for the distance function (2.4) the estimate from above

$$
d(R) \leq C_{1} \psi^{-1}(R) \quad(\underline{R} \leq R<\infty)
$$

with some constants $C_{1}>0$ and $\underline{R} \geq 0$. This implies an estimate from above for the error function of form

$$
f(\alpha)=\left\|x_{\alpha}-x_{0}\right\| \leq C_{2} \varrho^{-1}(\sqrt{\alpha})
$$

with some constant $C_{2}>0$ and for sufficiently small positive $\alpha$.

Proof. The hypotheses of the proposition ensure that the standing assumptions of [10] are satisfied with $\varrho_{1}(t)=\varrho(t)$ and $\varrho_{2}(t)=t$. As formulated in the proof of Theorem 1 in [10] and essentially based on Lemma 2 of [10] it can be seen that there exist constants $C_{1}>0$ and $\underline{R} \geq 0$ such that (3.2) holds. Then we have from (2.6)

$$
f(\alpha) \leq C_{1} \psi^{-1}(R)+\frac{\sqrt{\alpha}}{2} R \leq C_{0}\left(\psi^{-1}(R)+\sqrt{\alpha} R\right)
$$

with $C_{0}=\max \left(C_{1}, 1 / 2\right)$ and sufficiently large $R \geq 0$. By setting $R=R(\alpha)=$ $\varrho^{-1}(\sqrt{\alpha}) / \sqrt{\alpha}$ we see that $R(\alpha) \rightarrow \infty$ as $\alpha \rightarrow 0$, since $t / \varrho(t) \rightarrow \infty$ as $t \rightarrow 0$. Now we have $\varrho(\sqrt{\alpha} R(\alpha))=\varrho\left(\varrho^{-1}(\sqrt{\alpha})\right)=\sqrt{\alpha}$ and hence

$$
R(\alpha)=\frac{\sqrt{\alpha} R(\alpha)}{\varrho(\sqrt{\alpha} R(\alpha))}=\psi(\sqrt{\alpha} R(\alpha)),
$$

which gives $\psi^{-1}(R(\alpha))=\sqrt{\alpha} R(\alpha)$. Consequently, we obtain for sufficiently small $\alpha>0$ 


$$
f(\alpha) \leq C_{0}\left(\psi^{-1}(R(\alpha))+\sqrt{\alpha} R(\alpha)\right)=2 C_{0} \sqrt{\alpha} R(\alpha)=2 C_{0} \varrho^{-1}(\sqrt{\alpha}) .
$$

This yields (3.3) with $C_{2}=2 C_{0}$ and proves the proposition.

Note that the range inclusion (3.1) characterizes the interplay between solution smoothness in a very general sense expressed by properties of the operator $G$ and the smoothing property of the forward operator $A$. The index function $\varrho$ measures that interplay. More details on the interplay of smoothing conditions for Tikhonov regularization are given in [3] and for a general linear regularization approach in [17].

\section{An Application to Non-Compact Multiplication Operators}

Now we are going to illustrate the situations of Examples 2 and 3 in Section 2 in the context of non-compact multiplication operators $A$. In this section we consider $X=Y=L^{2}(0,1)$ and specify $A$ as a multiplication operator

$$
[A x](t)=m(t) x(t) \quad(0 \leq t \leq 1)
$$

defined by a multiplier function $m \in L^{\infty}(0,1)$ with essential zeros such that $\mathcal{R}(A)$ is not closed. For simplicity, let us assume

$$
x_{0}(t)=1 \quad(0 \leq t \leq 1)
$$

in the following two situations. Then we can formulate the following results. For proofs we refer to [8].

Proposition 3. For the solution (4.1) of equation (1.1) and the multiplier function

$$
m(t)=t \quad(0 \leq t \leq 1)
$$

we have with some constant $\underline{R}>0$ an estimate of the form

$$
d(R) \leq \frac{\sqrt{2}}{R} \quad(\underline{R} \leq R<\infty)
$$

for the distance function (2.4) of the pure multiplication operator A.

The situation of Proposition 3 corresponds to the case $\gamma=\frac{1}{2}$ in Example 2 and yields $f(\alpha)=\mathcal{O}(\sqrt[4]{\alpha})$ implying the Hölder rate $\mathcal{O}(\sqrt[3]{\delta})$, which is order optimal in that situation.

Proposition 4. For the solution (4.1) of equation (1.1) and the multiplier function

$$
m(t)=\sqrt{t} \quad(0 \leq t \leq 1)
$$

we have with some constant $\underline{R}>0$ an estimate of the form

$$
d(R) \leq \exp \left(-\frac{1}{2} R^{2}\right) \quad(\underline{R} \leq R<\infty)
$$

for the distance function (2.4) of the pure multiplication operator A. 
Obviously, the situation of Proposition 4 corresponds to the case $c=\frac{1}{2}$ and $q=2$ in Example 3 and yields $f(\alpha)=\mathcal{O}\left(\sqrt{\left(\ln \frac{1}{\alpha}\right) \alpha}\right)$. For that situation our alternative approach does not provide us with the order optimal convergence rate $f(\alpha)=\mathcal{O}(\sqrt{\alpha})$. This is a drawback of the suggested method based on Lemma 1. By construction of this approach we cannot obtain higher order rates $f(\alpha)=\mathcal{O}\left(\alpha^{\mu}\right)$ with $\mu \geq \frac{1}{2}$. This is some kind of limitation for the presented technique.

\section{A New Theorem on Compact Operators and a Case Study Concerning Simple Integration}

In this section we focus on ill-posed equations (1.1) with compact operators $A$ which are characterized by the singular system $\left\{\sigma_{i} ; u_{i} ; v_{i}\right\}$ of $A$. Here $\sigma_{1} \geq \sigma_{2} \geq \ldots>0$ denote the ordered singular values of $A$ with $\sigma_{i} \rightarrow 0$ as $i \rightarrow \infty, A u_{i}=\sigma_{i} v_{i}$ and $A^{*} v_{i}=\sigma_{i} u_{i}$, where $\left\{u_{i}\right\} \subset X$ and $\left\{v_{i}\right\} \subset Y$ are the orthonormal eigensystems of $A^{*} A$ and $A A^{*}$, respectively. First we present a theorem which shows that the converse result on distance functions $d(R)$ for general operators $A$ formulated as Proposition 2.6 in [8] can be improved in case of compact $A$. As a consequence of this new theorem it becomes evident that possible decay rates of power type for $d(R) \rightarrow 0$ as $R \rightarrow \infty$ are completely determined by the asymptotic behaviour of the Fourier coefficients $\left\langle x_{0}, u_{i}\right\rangle$.

Theorem 1. Let the injective linear operator $A: X \rightarrow Y$ of equation (1.1) be compact with the singular system $\left\{\sigma_{i}, u_{i}, v_{i}\right\}$ and let, for given $y \in \mathcal{R}(A)$, the uniquely determined solution $x_{0} \in X$ of (1.1) satisfies the condition $x_{0} \notin \mathcal{R}\left(A^{*}\right)$. Then a general source condition of power type

$$
x_{0}=\left(A^{*} A\right)^{\frac{\eta}{2}} w \quad(w \in X)
$$

implies for every exponent $0<\eta<1$ the estimate

$$
d(R) \leq K / R^{\frac{\eta}{1-\eta}} \quad(0<R<\infty)
$$

with some constant $K>0$.

Proof. For $x_{0} \notin \mathcal{R}\left(A^{*}\right)$ based on the Lagrange multiplier method we have

$$
d(R)=\lambda(R)\left\|\left(A^{*} A+\lambda(R) I\right)^{-1} x_{0}\right\|,
$$

where $\lambda=\lambda(R)>0$ is for all $R>0$ the uniquely determined solution of equation

$$
R^{2}=\left\|A\left(A^{*} A+\lambda I\right)^{-1} x_{0}\right\|^{2} .
$$

As already mentioned in [8, Proof of Lemma 2.5] this gives the formula

$$
d(R)=f(\lambda(R)) .
$$

On the one hand from (5.1), (5.4) and Proposition 1, taking into account that $\varphi(t)=t^{\frac{\eta}{2}}$ is concave for all $0<\eta<1$, we immediately derive an estimate 


$$
d(R) \leq(\lambda(R))^{\eta / 2}\|w\|
$$

Because of the well-known equivalence

$$
x_{0} \in \mathcal{R}\left(\left(A^{*} A\right)^{\frac{\eta}{2}}\right) \Longleftrightarrow \sum_{i=1}^{\infty} \frac{\left\langle x_{0}, u_{i}\right\rangle^{2}}{\sigma_{i}^{2 \eta}}<\infty
$$

(see [4, Proposition 3.13]) we have from (5.1) that

$$
\sum_{i=1}^{\infty} \frac{\left\langle x_{0}, u_{i}\right\rangle^{2}}{\sigma_{i}^{2 \eta}}=\hat{C}<\infty
$$

and hence the estimate

$$
\left\|A\left(A^{*} A+\lambda I\right)^{-1} x_{0}\right\|^{2}=\sum_{i=1}^{\infty} \frac{\left\langle x_{0}, u_{i}\right\rangle^{2}}{\sigma_{i}^{2 \eta}} \frac{\sigma_{i}^{2(1+\eta)} \lambda^{1-\eta}}{\left(\sigma_{i}^{2}+\lambda\right)^{2}} \lambda^{\eta-1} \leq \hat{C} \lambda^{\eta-1},
$$

since $\frac{\sigma_{i}^{2(1+\eta)} \lambda^{1-\eta}}{\left(\sigma_{i}^{2}+\lambda\right)^{2}} \leq 1$ is a consequence of Young's inequality.

On the other hand, we easily see that $\lambda(R) \leq \hat{\lambda}(R)$ for the solution $\lambda(R)$ of equation (5.3) whenever $\lambda=\hat{\lambda}(R)>0$ denotes for all $R>0$ the uniquely determined solution of equation

$$
R^{2}=\hat{C} \lambda^{\eta-1}
$$

Then we have $\hat{\lambda}(R)=\hat{\hat{C}} R^{\frac{2}{\eta-1}}$ with $\hat{\hat{C}}=(\hat{C})^{\frac{1}{1-\eta}}$. Taking into account (5.5) this provides us with a constant $K>0$ such that (5.2) is valid.

Remark 1. As a complement to Theorem 1 we obtain from Example 2 in Section 2 that an estimate of the form (2.11) with some fixed $0<\gamma<1$ can only hold if $x_{0}$ satisfies source conditions of the form (5.1) for all $0<\eta<\gamma$. Namely, from (2.11) we had found an inequality of the form (2.12) which implies (5.2) for all $0<\eta<\gamma$ (see [19]). More precisely, for an element $x_{0} \in X$ with

$$
0<\gamma_{\text {sup }}:=\sup \left\{\gamma>0: \sum_{i=1}^{\infty} \frac{\left\langle x_{0}, u_{i}\right\rangle^{2}}{\sigma_{i}^{2 \gamma}}<\infty\right\}<1
$$

we have estimates (2.11) of the distance functions $d(R)$ if and only if $0<\gamma \leq \gamma_{\text {sup }}$, i.e., via the value $\gamma_{\text {sup }}$ there is a one-to-one correspondence between maximal power type decay rates of the distance function $d(R) \rightarrow 0$ as $R \rightarrow \infty$ and maximal power type decay rates of the error function $f(\alpha) \rightarrow 0$ as $\alpha \rightarrow 0$ of Tikhonov regularization.

In the remaining part of this section we are going to illustrate the theory by some case studies concerning the simple integration operator

$$
[A x](s)=\int_{0}^{s} x(t) d t \quad(0 \leq s \leq 1)
$$


mapping in $X=Y=L^{2}(0,1)$ with explicitly available singular values

$$
\sigma_{i}=\frac{1}{\pi\left(i-\frac{1}{2}\right)} \sim \frac{1}{\pi i} \sim i^{-1} \quad(i=1,2, \ldots)
$$

and eigensystems

$$
u_{i}(t)=\sqrt{2} \cos \left(\left(i-\frac{1}{2}\right) \pi t\right), v_{i}(t)=\sqrt{2} \sin \left(\left(i-\frac{1}{2}\right) \pi t\right) \quad(0 \leq t \leq 1) .
$$

Then the degree of ill-posedness of a corresponding equation (1.1), the solution of which corresponds to the problem of finding the first derivative, is one and the problem is mildly ill-posed (for more details see, e.g., [9]).

We return in the sequel to the specific source condition (2.5) which can be rewritten for the integration operator (5.8) as

$$
x_{0}(t)=\left[A^{*} v\right](t)=\int_{t}^{1} v(s) d s \quad(0 \leq t \leq 1)
$$

with $\|v\|_{L^{2}(0,1)} \leq R_{0}$. This is equivalent to $x_{0} \in H^{1}(0,1)$ with $\left\|x_{0}^{\prime}\right\|_{L^{2}(0,1)} \leq R_{0}$ and $x_{0}(1)=0$ requiring a smoothness and a boundary condition for the function $x_{0} \in L^{2}(0,1)$ to satisfy $(2.5)$.

Example 4. In this example for equation (1.1) with operator $A$ from (5.8) we are going to consider what happens with respect to the loss of convergence speed in the Tikhonov regularization if a solution $x_{0}$ satisfying (2.5) is perturbed by adding a nonzero constant function $c(t) \equiv c>0$ such that the homogeneous boundary condition $x_{0}(1)=0$ gets violated. We assume the specific situation

$$
x_{0}=A^{*} v+c, \text { where }\left\langle v, v_{i}\right\rangle \sim \sigma_{i} \text { and }\left\langle c, u_{i}\right\rangle \sim \sigma_{i} \text { for } c \neq 0 .
$$

Then in view of

$$
\left\langle x_{0}, u_{i}\right\rangle=\left\langle A^{*} v+c, u_{i}\right\rangle=\sigma_{i}\left\langle v, v_{i}\right\rangle+\left\langle c, u_{i}\right\rangle
$$

it is evident that

$$
\left\langle x_{0}, u_{i}\right\rangle \sim \begin{cases}\sigma_{i}^{2} & \text { if } c=0 \\ \sigma_{i} & \text { if } c \neq 0 .\end{cases}
$$

Moreover, we recall that the equivalence (5.6) is valid for all $\eta>0$. Now for $c=0$ we have

$$
\sum_{i=1}^{\infty} \frac{\left\langle x_{0}, u_{i}\right\rangle^{2}}{\sigma_{i}^{2 \eta}}<\infty \text { if and only if } \sum_{i=1}^{\infty} i^{2 \eta-4}<\infty
$$

which is equivalent to $0<\eta<\frac{3}{2}$ and $\gamma_{\text {sup }}=\frac{3}{2}$ in the sense of (5.7). Consequently we obtain in the unperturbed case $c=0$ the rate $f(\alpha)=\mathcal{O}\left(\alpha^{\eta / 2}\right)$ 
just for all $0<\eta<\frac{3}{2}$. For an appropriate a priori parameter choice $\alpha(\delta)$ we can get here $e(\alpha(\delta), \delta)=\mathcal{O}\left(\delta^{3 / 5}\right)$ for $x_{0}=A^{*} v$.

On the other hand, we have for $c \neq 0$ in the perturbed case

$$
\sum_{i=1}^{\infty} \frac{\left\langle x_{0}, u_{i}\right\rangle^{2}}{\sigma_{i}^{2 \eta}}<\infty \text { if and only if } \sum_{i=1}^{\infty} i^{2 \eta-2}<\infty
$$

which is equivalent to $0<\eta<\frac{1}{2}$ and $\gamma_{\text {sup }}=\frac{1}{2}$. This reduces the possible decay rates of the error function $f(\alpha)=\mathcal{O}\left(\alpha^{\eta / 2}\right)$ to the smaller interval $0<\eta<\frac{1}{2}$. Here, for an optimal a priori parameter choice $\alpha(\delta)$ we can obtain $e(\alpha(\delta), \delta)=\mathcal{O}\left(\delta^{\frac{1}{3}}\right)$. Hence for $x_{0}$ from (5.12) the convergence rate exponent jumps downwards whenever with $c \neq 0$ the condition (2.5) gets violated. However, the jump altitude does not depend on the absolute value of $c$. Because of Theorem 1 an analogous jump occurs for the maximal exponent in the power type decay rate of $d(R)$.
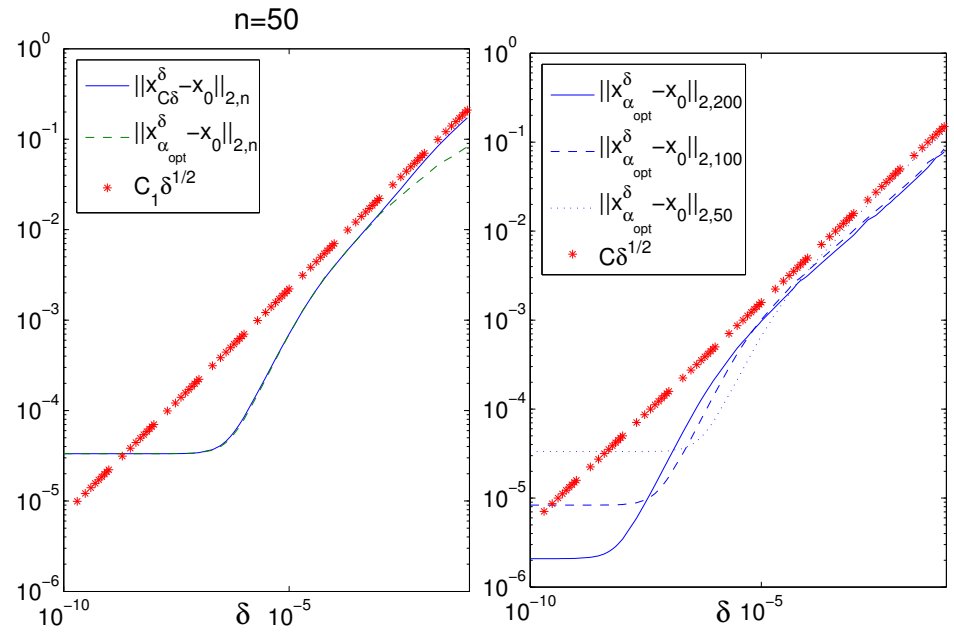

Figure 1. Error rates of Tikhonov regularization for solution $x_{0}(t)=(t-1)^{2}$.

For a case study (see [12]) illustrating Example 4 we consider the family of functions

$$
x_{0}(t)=(t-1)^{2}+\widetilde{c} t+c \quad(0 \leq t \leq 1)
$$

with arbitrary real constants $\widetilde{c}$ and $c$, where we can compute the inner products $\left\langle x_{0}, u_{i}\right\rangle$ explicitly as

$$
\left\langle x_{0}, u_{i}\right\rangle=\sqrt{2}\left((\widetilde{c}+c) \sigma_{i}(-1)^{i+1}+(2-\widetilde{c}) \sigma_{i}^{2}-2 \sigma_{i}^{3}(-1)^{i+1}\right) \quad(i=1,2, \ldots)
$$

with $\sigma_{i}$ from (5.9) and $u_{i}$ from (5.10). 
If we discretize the operator equation (1.1) with $A$ from (5.8) in an appropriate way by a linear system of algebraic equations with a system matrix of dimension $n \times n$ and use the Tikhonov regularization for the discrete analogue of (1.1) with solution (5.13) and $\widetilde{c}=c=0$, then we have $\left\langle x, u_{i}\right\rangle \sim \sigma_{i}^{2}$ and can check the convergence rate behaviour by a numerical study. Using doublelogarithmic scales in Figure 1 we show some results of this case $\widetilde{c}=c=0$, where discrete $L^{2}$ norms $\|\cdot\|_{2, n}$ in the $n$-dimensional Euclidean vector space were used to measure the regularization errors.

In the left part of Figure 1 we compare for dimension $n=50$ the total errors $e(\alpha(\delta), \delta)$ of the Tikhonov regularization for an a priori parameter choice $\alpha(\delta)=C \delta$ (solid line) and for an optimal parameter choice (dashed line). The star-shaped line indicates as a benchmark the rate $\mathcal{O}(\sqrt{\delta})$. It is shown that the optimal rate $e(\alpha(\delta), \delta)=\mathcal{O}\left(\delta^{3 / 5}\right)$ only takes place in the mid part of the figure, whereas for larger $\delta$ the error seems to behave more like $\mathcal{O}(\sqrt{\delta})$. On the other hand, we can see that for very small $\delta$ the discretization errors dominate the behaviour and they lead to a constant minimal error level. The right part of this figure clearly shows that this minimal error level as expected decreases with growing dimension $n$ of discretization.
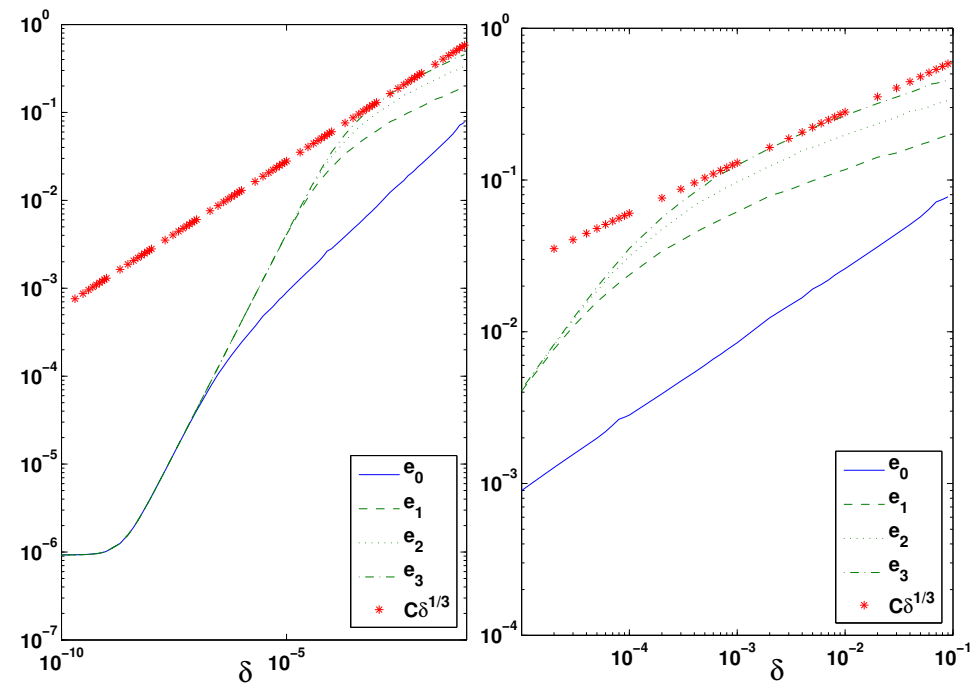

Figure 2. Errors for solutions $x_{j}(t)=(t-1)^{2}+j t(j=0,1,2,3)$ and $n=300$.

In Figure 2 we present results of another case of this study, where the functions $x_{j}(t)=(t-1)^{2}+j t(j=0,1,2,3)$ as perturbations of $(t-1)^{2}$ belong to the family (5.13) with $\widetilde{c}=j$ and $c=0$. We denote by $e_{j}$ the errors $e\left(\alpha_{o p t}(\delta), \delta\right)$ of the discrete Tikhonov regularization for solutions $x_{j}$. The star-shaped line with rate $\mathcal{O}\left(\delta^{1 / 3}\right)$ serves here as benchmark. One can 
see that the perturbations in particular influence the results for $\delta$ which are not too small and were zoomed in the right part of the figure. For $\delta>10^{-4}$ we see a significantly smaller decay rate of the errors $e_{1}, e_{2}$ and $e_{3}$ compared to the unperturbed case $e_{0}$.

The case study confirms that theoretically expected convergence rate jumps between the perturbed and the unperturbed case also occur if we consider a discrete version of Tikhonov regularization. However, for $\delta$ small enough the discretization error clearly dominates all other effects.

\section{On Distance Functions with Alternative Benchmarks}

By construction of $d(R)$ in (2.4) the rates of $f(\alpha) \rightarrow 0$ as $\alpha \rightarrow 0$, which can be obtained by our distance function method, are slower than $\mathcal{O}(\sqrt{\alpha})$ (cf. Example 2 in Section 2). Hence the corresponding convergence rates in the case of noisy data are slower than $\mathcal{O}(\sqrt{\delta})$ and hence there is a considerable gap to the saturation rate $\mathcal{O}\left(\delta^{2 / 3}\right)$ of the Tikhonov regularization. In order to close this gap, we can replace the specific source condition (2.5) as benchmark for the distance function $d(R)$ by a general source condition (2.1) with some index function $\varphi$. Then we have to consider the associated modified distance function

$$
\widetilde{d}(R):=\inf \left\{\left\|x_{0}-\varphi\left(A^{*} A\right) w\right\|: w \in X,\|w\| \leq R\right\}
$$

with a new benchmark. Here, $\widetilde{d}(R)$ measures the violation of $x_{0}$ with respect to the shifted benchmark. Based on formula (2.2) an analogue of Lemma 1 holds whenever one of the hypotheses of Proposition 1 concerning $\varphi$ is satisfied.

Lemma 2. Let $\varphi(t)\left(0 \leq t \leq\|A\|^{2}\right)$ be an index function that satisfies one of the requirements (a) - (e) in Proposition 1 with the corresponding constant $K \geq 1$. Then we obtain for the distance function (6.1) the error function estimate of the Tikhonov regularization

$$
f(\alpha)=\left\|x_{\alpha}-x_{0}\right\| \leq \widetilde{d}(R)+K \varphi(\alpha) R
$$

for all $\alpha>0$ and $R \geq 0$.

Proof. Let $w \in X$ with $\|w\| \leq R$. Then based on formula (1.2) and formula (2.2) we can estimate by the triangle inequality as follows:

$$
\begin{aligned}
f(\alpha)= & \| \alpha\left(A^{*} A+\alpha I\right)^{-1} x_{0}-\alpha\left(A^{*} A+\alpha I\right)^{-1} \varphi\left(A^{*} A\right) w \\
& +\alpha\left(A^{*} A+\alpha I\right)^{-1} \varphi\left(A^{*} A\right) w \| \\
\leq & \left\|\alpha\left(A^{*} A+\alpha I\right)^{-1}\left(x_{0}-\varphi\left(A^{*} A\right) w\right)\right\|+\left\|\alpha\left(A^{*} A+\alpha I\right)^{-1} \varphi\left(A^{*} A\right) w\right\| \\
\leq & \alpha\left\|\left(A^{*} A+\alpha I\right)^{-1}\right\|\left\|x_{0}-\varphi\left(A^{*} A\right) w\right\|+\left\|\alpha\left(A^{*} A+\alpha I\right)^{-1} \varphi\left(A^{*} A\right) w\right\| \\
\leq & \alpha \frac{1}{\alpha}\left\|x_{0}-\varphi\left(A^{*} A\right) w\right\|+K \varphi(\alpha)\|w\| \leq\left\|x_{0}-\varphi\left(A^{*} A\right) w\right\|+K \varphi(\alpha) R .
\end{aligned}
$$


Since the inequality

$$
f(\alpha) \leq\left\|x_{0}-\varphi\left(A^{*} A\right) w\right\|+K \varphi(\alpha) R
$$

also is true if we substitute $\left\|x_{0}-\varphi\left(A^{*} A\right) w\right\|$ by

$$
\inf \left\{\left\|x_{0}-\varphi\left(A^{*} A\right) w\right\|: w \in X,\|w\| \leq R\right\},
$$

we obviously have (6.2). This proves the lemma.

In particular for the interesting limit case $\varphi(t)=t$ we have the estimate

$$
f(\alpha) \leq \widetilde{d}(R)+\alpha R
$$

for all $\alpha>0$ and $R \geq 0$. Along the lines of Examples $1-3$ this may yield all convergence rates which are slower than $f(\alpha)=\mathcal{O}(\alpha)$ provided that a sufficiently rapid decay of $\widetilde{d}(R) \rightarrow 0$ as $R \rightarrow \infty$ occurs. Then for noisy data convergence rates can be derived which are arbitrarily close to the saturation rate $\mathcal{O}\left(\delta^{2 / 3}\right)$.

In the forthcoming work of the first and the second authors and coauthors we will study the consequences of Lemma 2 for examples and will extend the applications presented in Sections $3-5$ to the case of general benchmark functions $\varphi$ in (6.1). However, we should mention that the only consideration of the limit case $\varphi(t)=t$ in (6.1) with inequality (6.3) does not make superfluous all the studies on $\varphi(t)=\sqrt{t}$ and distance function (2.4) presented in this paper. Namely, for example a range inclusion

$$
\mathcal{R}(\varrho(G)) \subset \mathcal{R}\left(A^{*} A\right),
$$

which then would be required instead of (3.1), is more difficult to satisfy than (3.1), because the range $\mathcal{R}\left(A^{*} A\right)$ can be essentially smaller than the range $\mathcal{R}\left(A^{*}\right)$ under consideration in this paper.

\section{Acknowledgement}

The authors would like to thank Professor Heinz W. Engl (Linz, Austria) for his valuable suggestions concerning the compact case in the context of a talk given by the third named author in Linz. Those suggestions gave us motivation to formulate and to prove Theorem 1. 


\section{References}

[1] A.B. Bakushinksy and M.Yu. Kokurin. Iterative Methods for Approximate Solutions of Inverse Problems. Springer, Dordrecht, 2004.

[2] J. Baumeister. Stable Solution of Inverse Problems. Vieweg, Braunschweig, 1987.

[3] A. Boettcher, B. Hofmann, U. Tautenhahn and M. Yamamoto. Convergence rates for Tikhonov regularization from different kinds of smoothness conditions. Applicable Analysis, 85, 2006. In press. Available electronically as DOI: $10.1080 / 00036810500474838$.

[4] H.W. Engl, M. Hanke and A. Neubauer. Regularization of Inverse Problems. Kluwer, Dordrecht, 1996.

[5] M. Freitag and B. Hofmann. Analytical and numerical studies on the influence of multiplication operators for the ill-posedness of inverse problems. Journal of Inverse and Ill-Posed Problems, 13, 123-148, 2005.

[6] C.W. Groetsch. The Theory of Tikhonov Regularization for Fredholm Integral Equations of the First Kind. Pitman, Boston, 1984.

[7] B. Hofmann. Approximate source conditions in regularization and an application to multiplication operators. In: R. Čiegis(Ed.), Mathematical Modelling and Analysis 2005. Proc. 10th Int. Conf. MMA2005 \& CMAM2, Trakai, Lithuania, 2005, Technika, Vilnius, 29 - 34, 2005.

[8] B. Hofmann. Approximate source conditions in Tikhonov-Phillips regularization and consequences for inverse problems with multiplication operators. Mathematical Methods in the Applied Sciences, 29, 351-371, 2006.

[9] B. Hofmann and L. von Wolfersdorf. Some results and a conjecture on the degree of ill-posedness for integration operators with weights. Inverse Problems, 21, $427-433,2005$.

[10] B. Hofmann and M. Yamamoto. Convergence rates for Tikhonov regularization based on range inclusions. Inverse Problems, 21, 805-820, 2005.

[11] T. Hohage. Regularization of exponentially ill-posed problems. Numerical Functional Analysis and Optimization, 21, 439 - 464, 2000.

[12] K. Krumbiegel. On the influence of approximate source conditions on accuracy and convergence rates of regularized solutions. Faculty of Mathematics, Chemnitz University of Technology, Chemnitz, Germany, 2005. Diploma thesis (in German).

[13] J. Locker and P.M. Prenter. Regularization with differential operators - first part: General Theory. Journal of Mathematical Analysis and Applications, 74, $504-529,1980$.

[14] P. Mathé and S.V. Pereverzev. Moduli of continuity for operator valued functions. Numer. Funct. Anal. Optim., 23, 623 - 631, 2002.

[15] P. Mathé and S.V. Pereverzev. Geometry of linear ill-posed problems in variable Hilbert scales. Inverse Problems, 19, $789-803,2003$.

[16] P. Mathé and S.V. Pereverzev. Regularization of some linear ill-posed problems with discretized random noisy data. Mathematics of Computation, 75, 2006. In press.

[17] M.T. Nair, S.V. Pereverzev and U. Tautenhahn. Regularization in Hilbert scales under general smoothing conditions. Inverse Problems, 21, 1851 - 1869, 2005.

[18] M.T. Nair, E. Schock and U. Tautenhahn. Morozov's discrepancy principle under general source conditions. Zeitschrift fuer Analysis u. Anwendungen, 22, $199-214,2003$.

[19] A. Neubauer. On converse and saturation results for Tikhonov regularization of linear ill-posed problems. SIAM J. Numer. Anal., 34, 517 - 527, 1997. 
[20] U. Tautenhahn. Optimality for ill-posed problems under general source conditions. Numerical Functional Analysis and Optimization, 19, 377 - 398, 1998.

[21] A.N. Tikhonov and V.Y. Arsenin. Solutions of Ill-Posed Problems. Wiley, New York, 1977. 